\title{
Effects of drought stress on soil bacteriall community in the West foot of Daxing'an Mountains
}

\author{
Shuli Wei ${ }^{1,2}$, Jing Fang ${ }^{1,2}$,Gongfu Shi ${ }^{1,2}$, Yuchen $\mathrm{Cheng}^{2}$, Jianhui $\mathrm{Wu}^{1,2}$,Erhu $\mathrm{Su}^{3}$, Zhixiong Liu ${ }^{3}$, Zhanyuan $\mathrm{Lu}^{1,2, *}$, \\ Xiaoqing Zhao ${ }^{1,2, *}$ \\ ${ }^{1}$ School of Life Science, Inner Mongolia University, China; Key Laboratory of Herbage \& Endemic Crop Biotechnology, Ministry of \\ Education, China \\ ${ }^{2}$ Inner Mongolia Academy of Agricultural \& Animal Husbandry Sciences/ Inner Mongolia Conservation Tillage Engineering \\ Technology Research Center/ Inner Mongolia Key Laboratory of Degradation Farmland Ecological Restoration and Pollution Control, \\ China \\ ${ }^{3}$ Inner Mongolia Academy of Agricultural \& Animal Husbandry Sciences Corn Research Institute, China
}

\begin{abstract}
Global warming poses a serious threat to agriculture and natural systems, in part because of the change of soil moisture content, which changes soil microbial communities and ecological processes. Soil water content is the main factor limiting the growth of plants in soil. Microbial communities rely on soil water to complete their activities, and reveal the changes of underground microbial communities under different soil moisture content, which will help us to further understand the potential impact of climate change on soil ecosystem. To investigate the soil bacterial community structure, we established experiment indoor in the West foot of Daxing'an Mountains with manipulative water content treatments consisting of $20 \%, 15 \%, 10 \%, 5 \%, 0 \%$. Results showed that bacterial community composition varied significantly with altered drought stress, but community richness did not. The relative abundance of Actinobacteria increased with the increase of drought stress, Proteobacteria, Acidobacteria and Gemmatimonadota decreased with the increase of drought stress, actinobacteria was more likely to accumulate or maintain stable under drought stress, bacterial communities can responding directly to changes in soil moisture.
\end{abstract}

\section{Introduction}

Global climate change may have considerable impacts on hydrological cycles worldwide, which may result in increased precipitation, higher evaporation rates, and the uneven distribution of rainfall[1]. Some regions may witness significant changes in the timing of dry and wet seasons, which might trigger increases in both droughts and floods[2]. Global warming poses serious threats to agricultural and natural systems, partly through its capacity to alter soil microbial communities and ecological processes due to variations in the content of soil water content[3]. Immense diversity of microorganisms that live belowground contributes significantly to shaping aboveground biodiversity and the functioning of terrestrial ecosystems[4]. Zhang et al analyzed the distribution and variation of temperature and precipitation in the Western foot of Daxing'an Mountains of China in $1960-2015$, over the past 56 years, it has shown a trend of warming and drying[5]. Consequently, uncovering the effect of drought stress on the microbial community composition in the Western foot of Daxing'an Mountains will considerably enhance our understanding of the potential impact of climate change on arid ecosystems and their functions.
Soil microorganisms are active factors in the soil ecosystem. They play an important role in soil formation and development, soil organic matter decomposition, material and energy input, nutrient conversion, and fertility evolution. They are important links in the material cycle ecological chain[6,8]. The Soil water content is one of critical environmental factors that influence microbial community structure and function [7], and the microbial community rely on soil moisture to complete their activities and life spans[6]. A high SWC decreases microbial degradation rates because of low oxygen supply; a low soil water content decreases both microbial mobility and microbial degradative activity by reducing the diffusion of soluble substrates[9]. Researchers have observed increased rainfall might alter the elemental compositions of karst soils, and bacteriall communities are likely to be more sensitive to variations in soil moisture in contrast to their fungal counterparts[10]. Microcosm-based experiments with tropical or subtropical soil have shown that soil water content exerts more significant effects on bacteriall diversity and structure compared to Soil temperature [11-12]. Soil bacteriall communities can provide biologically relevant insights on the impacts of land use on soil ecosystems[13]. A diverse adaptation strategy of

\footnotetext{
*Corresponding author: lzhy281@163.com; zhaoxq204@163.com.
} 
different bacteriall groups. Actinobacterial could play an important role in nitrogen fixation, Bacillus and Pseudomonas can form endospores under drought stress, thereby resisting the variation caused by altered precipitation regimes[14], the phylogenetic shifts in the composition of soil microbial communities are correlated with community function $[15,16]$. How the bacteriall community structure and potential function without plants respond to water changes is poorly understood.

For this study, we conducted a controlled soil water content experiment in 5 gradient about the black soil of Daxing'an Mountains in China. Control variable method, high-throughput sequencing and bioinformatics were combined. The main aim is to determine the effects of the drought stress increased on soil bacteriall communities and potential functions, to explore the direct effect of water content on soil microbial community.

\section{Materials and methods}

\subsection{Experimental design}

In the scientific observation and Experiment Station of soil management and ecological restoration of tnihe, chenbalhu banner, Hulunbuir City, the West foot of Daxinganling Mountains, $160 \mathrm{~kg}$ soil was taken, mixed evenly, and dried in the room.

Five treatments were set up in the experiment. The relative water content of soil was $20 \%$ (water needed for normal plant growth), $15 \%, 10 \%, 5 \%$ and $0 \%$ respectively. Each treatment was repeated three times. The soil after shade drying was divided into 15 parts, each $10 \mathrm{~kg}$. The soil was placed in a plastic flowerpot of $48.4 \mathrm{~cm} \times 32.2 \mathrm{~cm} \times 25.7 \mathrm{~cm}$. The soil without water was marked W0. The other groups were marked W20, w15, $\mathrm{W} 10$ and W5. After 72 hours of cultivation in gradient incubator (lt-36vl) (the preliminary experiment found that the soil microbial community would maintain a new stable state after 72 hours of cultivation), the soil was cultured in light for 16 hours a day and in dark for 8 hours, and water was added every 8 hours. Samples were taken after cultivation, the three repeated samples were evenly stirred, screened with $0.5 \mathrm{~mm}$ sterilized nylon sieve, and the screened soil was put into five $5 \mathrm{ml}$ centrifuge tubes, The DNA was labeled, frozen in liquid nitrogen and stored in a refrigerator at $-80^{\circ} \mathrm{C}$ for DNA isolation.

Relative water content $(\%)=\left(\right.$ Weight $_{\text {wet }}-$ Weight $\left._{\text {dry }}\right)$ $/$ Weight $_{\text {wet }} \times 100 \%$.

\section{2 $16 \mathrm{~S}$ metabarcoding and bioinformatics}

Total soil DNA was extracted from replicate samples $(\mathrm{n}=$ 5) by using DNeasy ${ }^{\circledR}$ PowerSoil ${ }^{\circledR}$ Pro Kit. After monitoring the DNA integrity and purity on $1 \%$ agarose gels, PCR was carried out using the bacterial-specific primer pair 515F (GTGCCAGCMGCCGCGGTAA)and 806R (GGACTACHVGG GTWTCTAAT) with a 6 bp error correcting barcode unique to each sample. DNA was amplified and then agarose gel was used to detect the quality of DNA. Then the concentration of PCR product was mixed with equal concentration, and then the GeneJET gel Recovery Kit (Thermo Scientific) and 1 Gene JET were used. The PCR product was purified by agarose gel electrophoresis with TAE concentration of $2 \%$, and the target band (size of 400 450bp sequence) was recovered by tapping. The library was constructed by using truseq DNA PCR free library preparation kit of illustra company. After qubit quantification and library test, the constructed library was sequenced by novaseq 6000 (Beijing Nuohe Zhiyuan bioinformatics Co., Ltd.).

\subsection{Statistical analysis}

The alpha diversity of samples was calculated with alpha_diversity.py in QIIME with the lowest number of sequences of all samples. The difference was analyzed by Kruskal Wallis h test, and Simpson and ACE index were calculated to characterize the bacterial community $\alpha$ Diversity. Based on Bray_Curtis distance matrix PCA was used to analyze the bacterial community structure.Lefse multi-level species difference discriminant analysis was used to indicate species, and nonparametric Kruskal Wallis (kw) sum rank test was used to detect species richness differences among different groups, and significant species were obtained and the significance difference of species in multiple groups of samples is analyzed.SigmaPlot was used to fit the quadratic function of different species with soil water content.

\section{Rresult}

\subsection{Community composition of bacterial in soils of in the West foot of Daxing'an Mountains}

A total of bacterial sequences from the 15 soil samples were obtained from a total of 1167155 sequences. The amount of sequencing data is enough(Fig.1A). When grouped at the level of $97 \%$ similarity, 3941 bacterial OTUs were formed. At the lower level of classification, there were 37Class, 104 orders, 265 families, 426 genera, and 1512 species found in the West foot of Daxing'an Mountains soils. Across all samples, the dominant soil bacterial phyla were Actinobacterial(31.94\%), Proteobacterial(26.70\%), Acidobacterial(10.22\%), Bacteroidota(8.93\%), Gemmatimonadota(6.92\%), Chloroflexi(6.64\%), Verrucomicrobiota(2.27\%), Myxococcota(1.82\%), and Planctomycetes $(1.10 \%)$, which collectively accounted for $96.08 \% \sim 97.18 \%$ of all taxon sequences(Fig.1B).

\subsection{Dissimilarity of bacterial communities between different drought stress}

We used the ACE index to estimate the richness of bacterial communities, and the Simpson index to estimate the diversity of bacterial communities in the 5 different water content. The results indicated that 
richness of the bacterial communities was no significant change(Fig.1C), but the diversity followed the different trend. The highest was for the W0, followed by W5, $\mathrm{W} 10, \mathrm{~W} 15$, and $\mathrm{W} 20$. The diversity of samples belonging to the W0 exhibited a greater variation range significantly than for the other soil water content, reflecting an association between community structure and soil water content, with the increase of drought stress, the species diversity increased(Fig.1D).
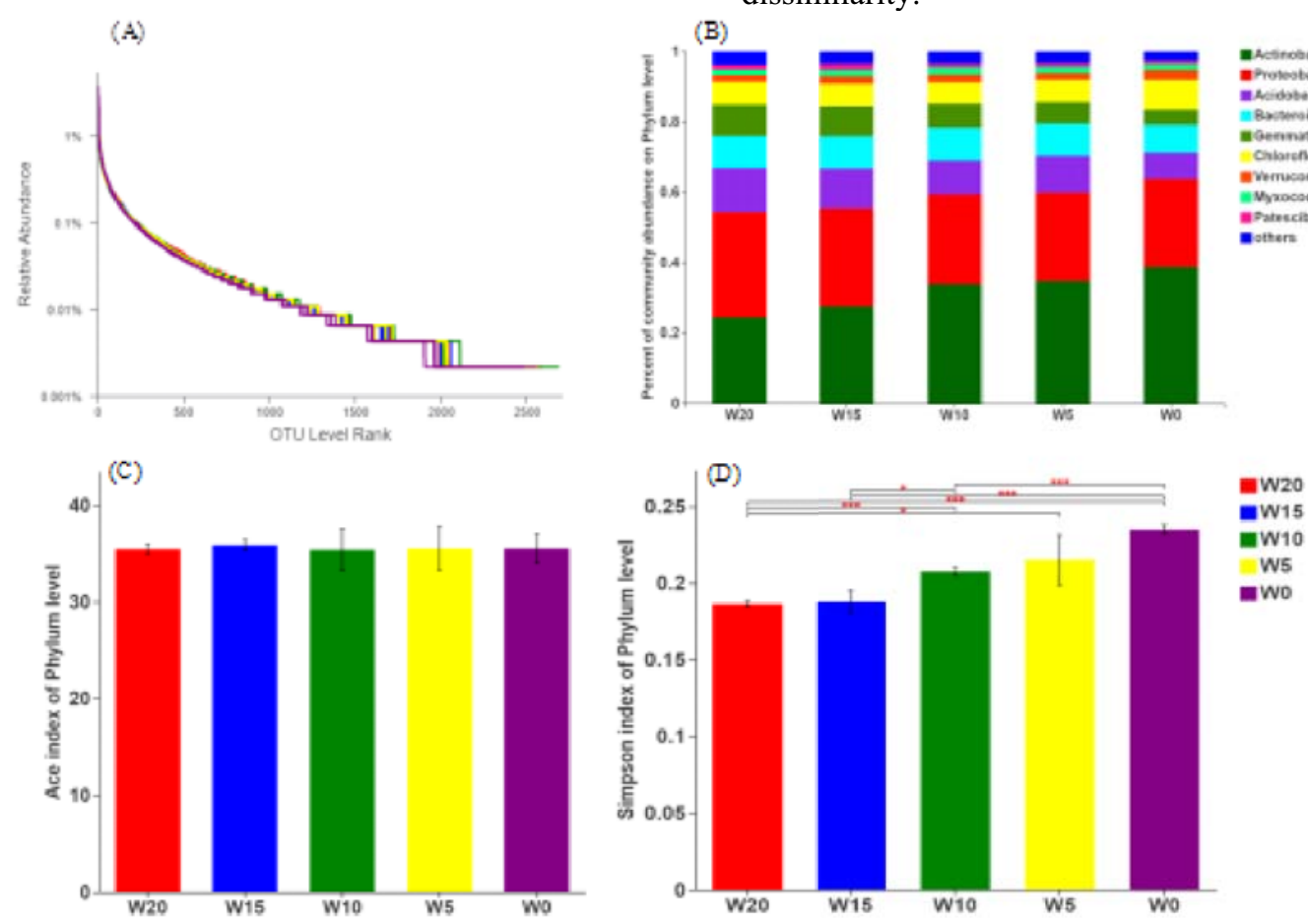

Fig.1. Rank-Abundance curves(A) and Soil bacterial community abundance at the phylum level(B) and the Significance analysis of

ACE(C) Simpson index(D) index under different soil water content. at the phylum level

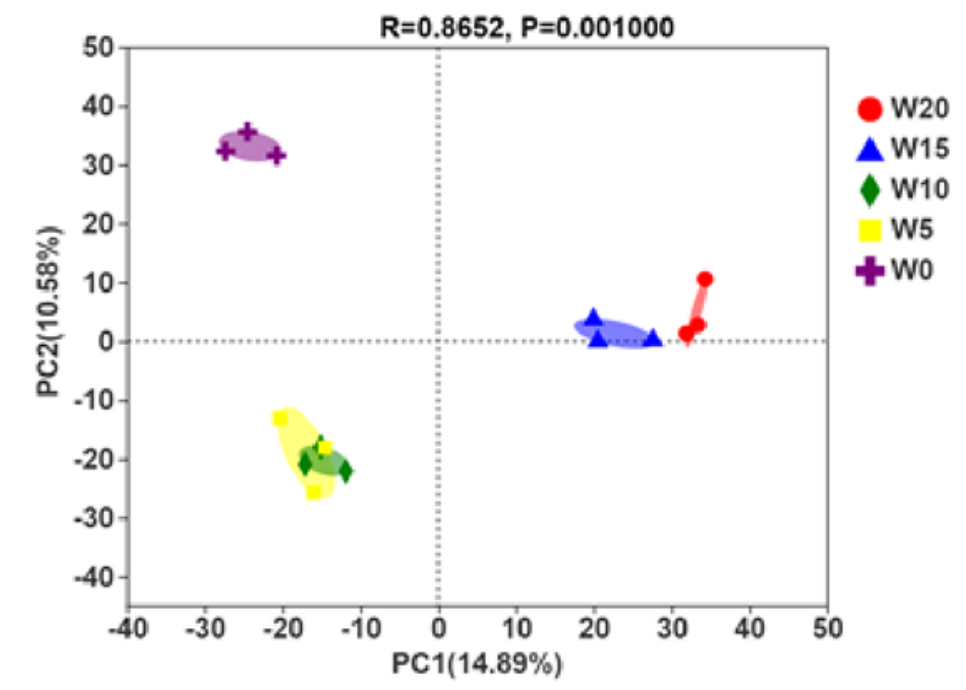

Fig. 2. Principal component analysis (PCA) plots of soil bacterial community composition on the basis of Bray_Curtis distance.

Using LefSe analysis, we obtained bacterial biomarkers from phyum to species for each soil water

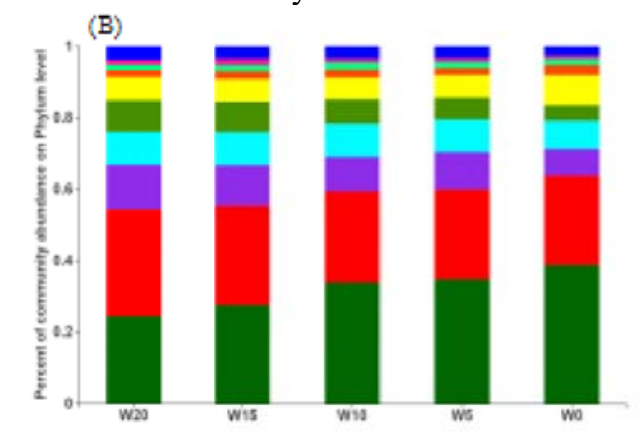

Dissimilarities in the bacterial communities from the 5 soil water content were identified with a PCA biplot(Fig.2). Notably, the composition of the bacterial communities was significantly affected by the soil water content(ANOSIM test, $\mathrm{R}=0.8652, \mathrm{p}=0.001$ ). Comparing the differentences of bacterial communities between each soil water content, indicated that W5 and W10 were the most similar, whereas W0 compared with other soil water content exhibited the largest dissimilarity.

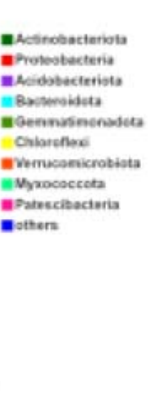

W20

W5

wo

content. There were 19 biomarkers identified in W20, 11 in W15, two in W10, two in W5 and 28 in W0(Fig.3). 


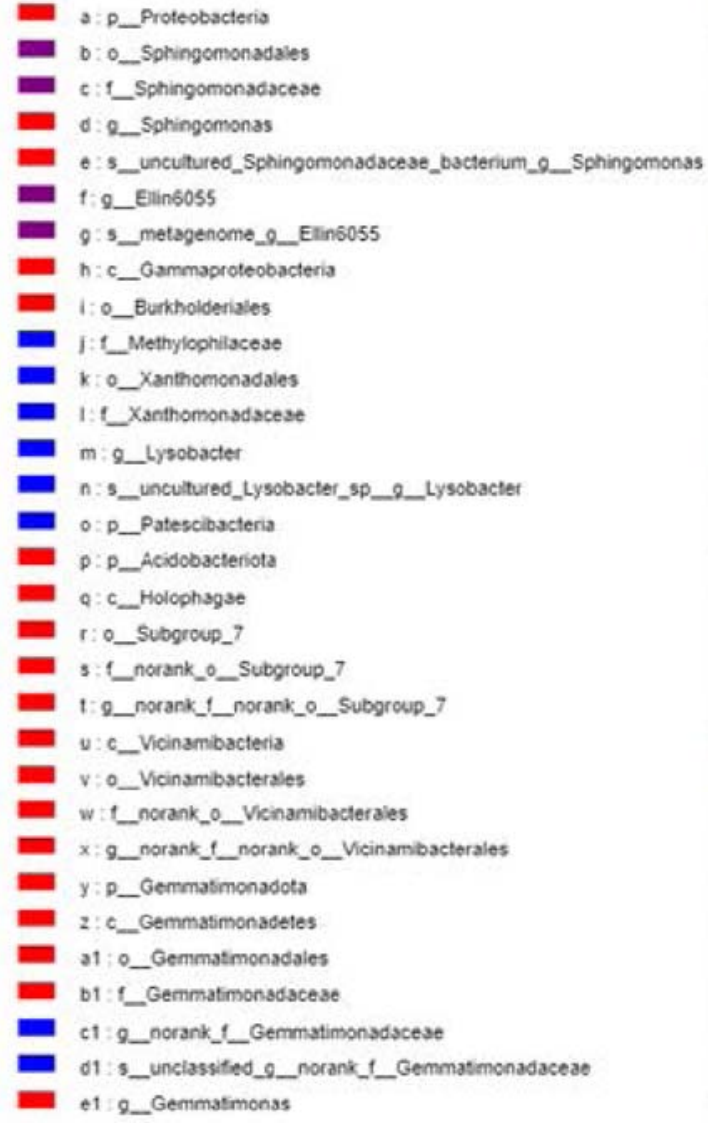

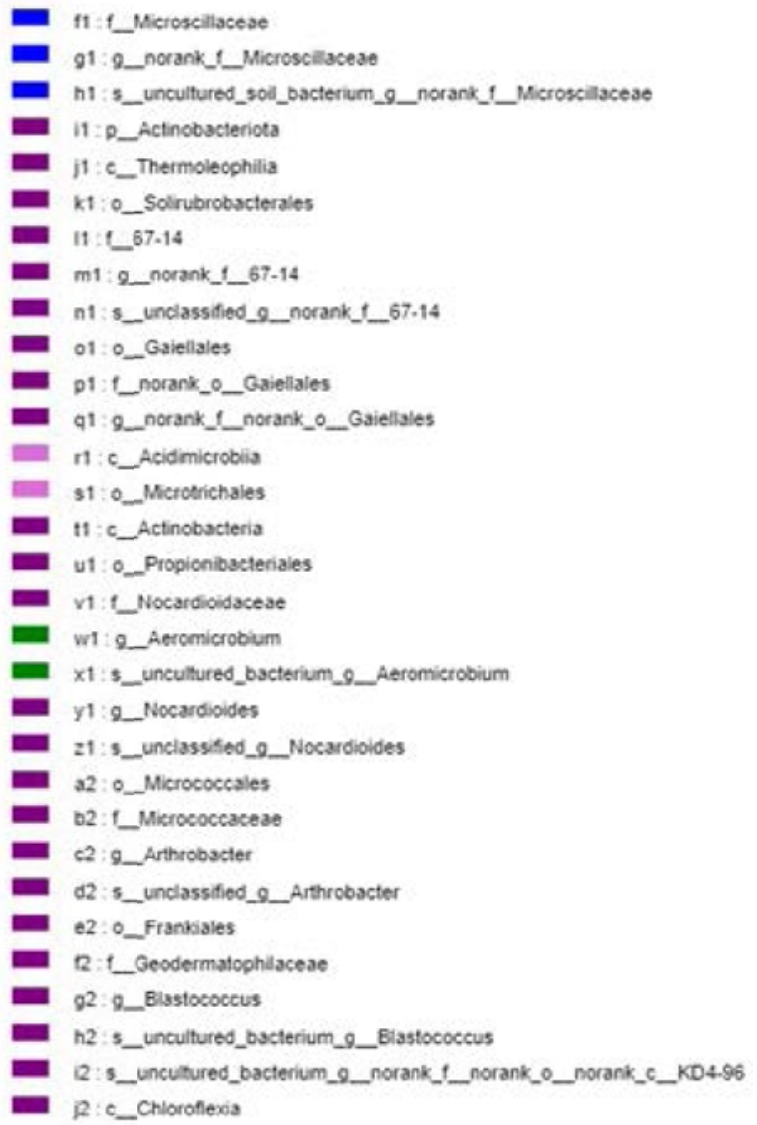

Fig.3.Taxonomic composition of bacterial community from five soil water content.

\subsection{Changes of bacterial community under different drought stresses}

The significance test results demonstrated that altered soil water content soil water content significantly affected the abundance of Actinobacteria, Proteobacteria, Acidobacteria and Gemmatimonadota from the top 10 phyla of abundance(Table.1).

The significance analysis of the top 27 genera (Relative aboundance $>1 \%$ ) with relative abundance showed that soil water content had a significant effect on 16 genera, and 16 genera belonged to 6 different phylum, which 8 belonged to Actinobacteria, 3 belonged to Proteobacteria, 2 belonged to Acidobacteria, gemmatimonadota and bacteroidota only have 1 respectively(Table.2). Among the 16 genera, the relative abundance of 8 genera of Actinobacteria and ellin6055 increased with the increase of drought stress, which they may belong to drought tolerant bacteria. The relative abundance of Lysobacter and g norank_ $f$ _ norank o vicinamibacteria decreased with the aggravation of drought stress that was sensitive to water content. With the increase of drought stress, the relative abundance of Sphingomonas, g_norank_f__Microscillaceae and g_norank_f__norank_o__norank_c_Gitt-GS-136 decreased first and then increased, and the adaptability was strong. They could adjust its adaptability with the change of soil water content; g_norank_f Microscillaceae increased first and then decreased with the increase of drought stress(Fig.5).

Table 1. Significant differences between groups in relative abundance of 10 most dominant soil bacterial phyla under altered soil water content

\begin{tabular}{lcc}
\hline Species name & P Value & Kruskal-Wallis H test \\
\hline Actinobacteriota & 0.020 & $*$ \\
Proteobacteria & 0.036 & $*$ \\
Acidobacteriota & 0.016 & $*$ \\
Bacteroidota & 0.453 & $\mathrm{~ns}$ \\
Gemmatimonadota & 0.012 & $*$ \\
Chloroflexi & 0.106 & $\mathrm{~ns}$ \\
Verrucomicrobiota & 0.264 & $\mathrm{~ns}$ \\
Myxococcota & 0.277 & $\mathrm{~ns}$ \\
Patescibacteria & 0.122 & $\mathrm{~ns}$ \\
Firmicutes & 0.271 & $\mathrm{~ns}$ \\
\hline
\end{tabular}

NOTE:"*" has significant difference at $\mathrm{P}<0.05$ level,and "ns" has no significant difference, the same as below. 

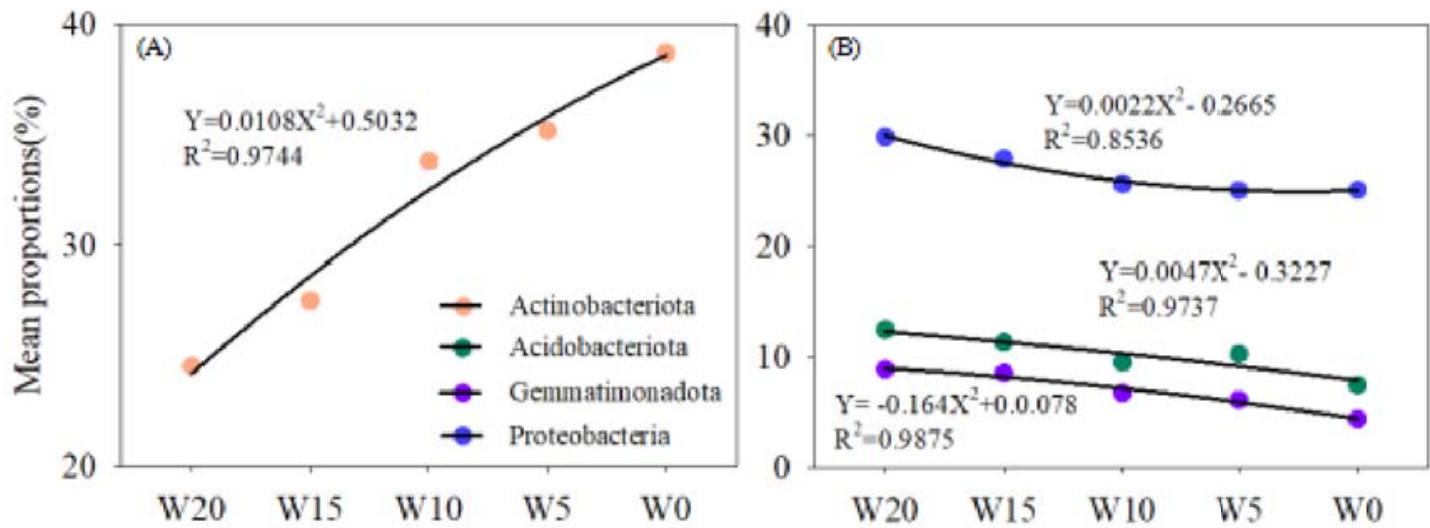

Fig.4. Variation trend of 4 phyla with soil water content

Table 2. Significant differences between groups in relative abundance of 25 most dominant soil bacterial genus under altered soil water content

\begin{tabular}{|c|c|c|c|}
\hline Phyum & Species name & P Value & Kruskal-Wallis $H$ test \\
\hline $\mathrm{p} \_$Actinobacteriota & $\mathrm{g} \_$norank_f_norank_o__Gaiellales & 0.0439 & * \\
\hline $\mathrm{p} \_$Actinobacteriota & $\mathrm{g} \_$norank_f_6 67-14 & 0.0285 & * \\
\hline $\mathrm{p} \_$Actinobacteriota & g__Blastococcus & 0.0273 & * \\
\hline $\mathrm{p} \_$Actinobacteriota & g_Arthrobacter & 0.0237 & * \\
\hline $\mathrm{p} \_$Actinobacteriota & g__Nocardioides & 0.0144 & * \\
\hline $\mathrm{p} \_$Actinobacteriota & $\mathrm{g} \_$norank_f_norank_o__Microtrichales & 0.0315 & * \\
\hline $\mathrm{p} \_$Actinobacteriota & g_norank_f_norank_o_norank_c__MB-A2-108 & 0.0388 & * \\
\hline $\mathrm{p} \_$Actinobacteriota & g_Aeromicrobium & 0.0273 & * \\
\hline p__Proteobacteria & g_Ellin6055 & 0.0166 & * \\
\hline p__Proteobacteria & g__Sphingomonas & 0.0181 & * \\
\hline p__Proteobacteria & g__Lysobacter & 0.0118 & * \\
\hline $\mathrm{p} \_$Acidobacteriota & g_norank_f_norank_o__Vicinamibacterales & 0.0125 & * \\
\hline $\mathrm{p} \_$Acidobacteriota & g__Bryobacter & 0.0203 & * \\
\hline p__ Gemmatimonadota & $\mathrm{g} \_$norank_f_Gemmatimonadaceae & 0.0255 & $*$ \\
\hline p__Bacteroidota & g_norank_f__Microscillaceae & 0.0255 & * \\
\hline $\mathrm{p} \_$Chloroflexi & g_norank_f_norank_o_norank_c__Gitt-GS-136 & 0.0404 & $*$ \\
\hline
\end{tabular}

In these four phyla, the relative abundance of Actinobacteria increased with the increase of drought stress. Compared with W20, the abundance of W15, W10, W5 and W0 increased respectively $12.06 \%$, $37.65 \%, 43.40 \%$ and $57.66 \%$, the highest abundance was in extreme drought; However, the abundance of Proteobacteria, acidobacteria an gemmatimonadota decreased with the increase of drought stress, indicating that actinobacteria was more likely to accumulate or maintain stable under drought stress(Fig.4).

\section{DISCUSSION}

\subsection{Dissimilarity of bacterial communities between different drought stress}

Our analyses indicate that soil water content significantly influence the composition of bacterial communities in the the West foot of Daxing'an Mountains. With the increase of drought stress increased species diversity and reached the maximum at W0. some studies have found that microbial community structure[17] and richness[18]change in response to increased precipitation, whereas other works did not observe substantial effects on microbial community 
composition[19-20]. Maybe the factor of water alter soil microbialcommunities by changing soil physical and chemical properties, nutrient cycling[21-23].

\subsection{A diverse adaptation strategy of different bacterial groups}
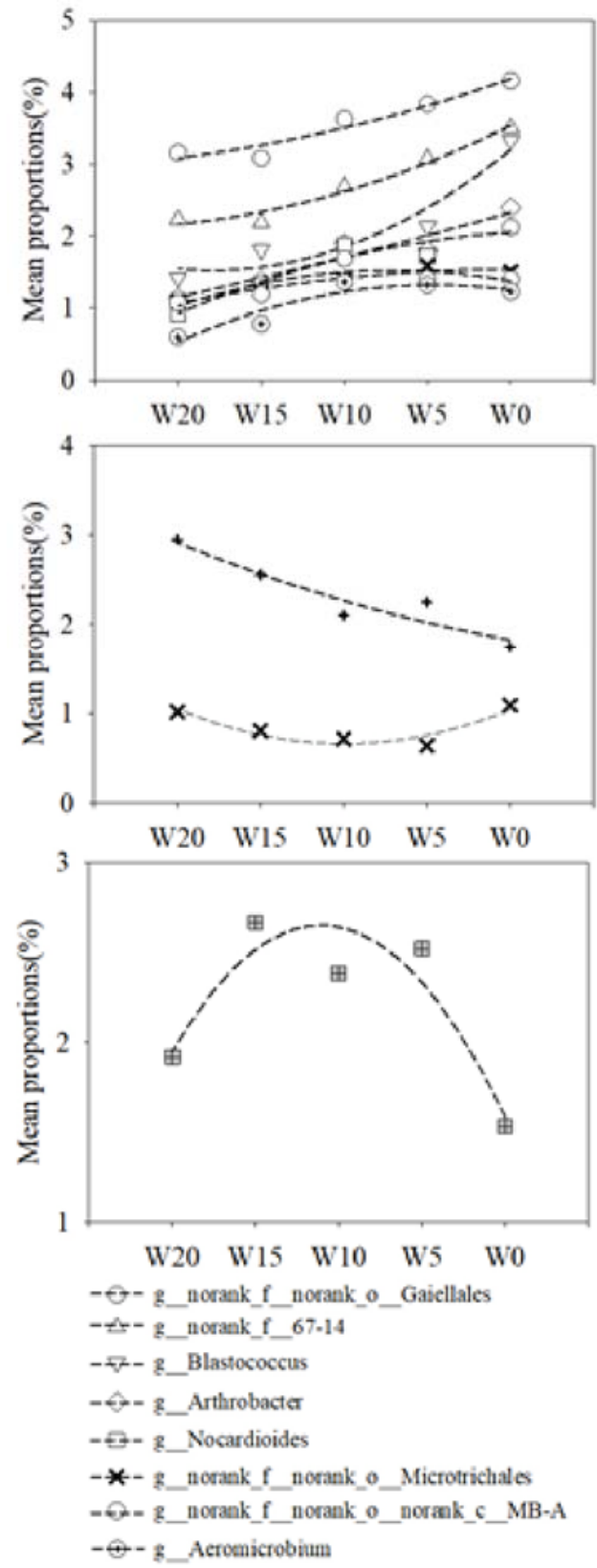

Four major response patterns were detected across all bacteriall samples[21], the first pattern was that resource-limited populations, some bacteriall increased in relative abundance with the increase in precipitation. By contrast, an opposite result was found which suggested that these genera belong to drought-tolerant populations. The third pattern showed that bacteriall genera were most abundant.
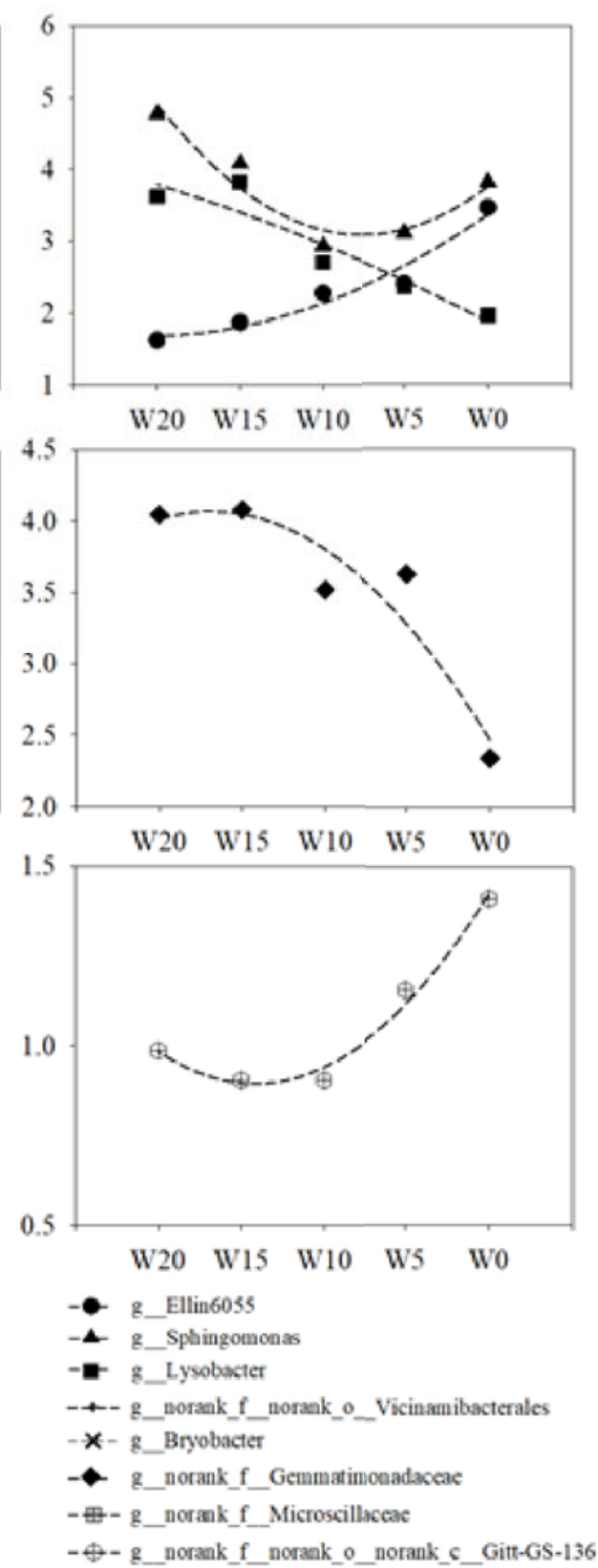

Fig.5. Variation trend of 16 genus with soil water content under different phyla

Consistent with the results of previous studies, but because there are no different plants and soil types, so the types of bacteria are different. The relative abundance of Proteobacteria, Acidobacteria and Gemmatimonadota decreased with the increase of drought stress. Actinobacteria were more likely to accumulate or remain stable under drought stress. Blastococcus, Arthrobacter, Nocardioides, Aeromicrobium bacteria belonged to Actinobacteria,
According to the trend analysis, they also had drought resistance characteristics, and the relative abundance increased with the increase of drought stress. Lysobacter and $g$ _norank_f_norank_o_vicina-mibacteria

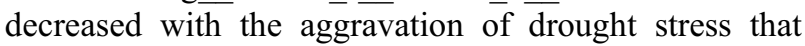
were sensitive to water content. Sphingomonas, $g$ _norank_f_Microscillaceae g__norank_f_norank_o_norank_c_Gitt-GS-136 
decreased first and then increased, and the adaptability was strong.

\subsection{Actinobacteria have a rich aboundance for under drought stress}

Actinobacteria are known for their metabolic potential of producing diverse secondary metabolites such as antibiotics. Actinobacteria play an important role in nitrogen cycling in both desert and cultivated farm ecosystems.Actinobacteria is associated with environmental factors, such as soil type[24]. They are important for the cycling of carbon, nitrogen, phosphorus, potassium, and several other elements in the soil[25]. Some members of the phylum

Actinobacteria may be able to live in an oligotrophic environment. Arocha-Garza [26] isolated 350 different strains from an oligotrophic desert oasis. The Actinobacteria can produce a variety of extracellular hydrolytic enzymes to degrade animal and plant residues, litter and other organic compounds in soil,enabling them to thrive in oligotrophic environments[27]. Our research indaceted that drought stress had a significant effect on soil actinobacteria. The highest abundance of actinobacteria was found in all the varieties, and its abundance increased with the increase of drought stress. After significance analysis and trend fitting, it was found that $50 \%$ of the 16 genera responding to drought stress belonged to actinobacteria. It can be enriched to cope with drought.in the natural precipitation. Lastly, relative abundance did not vary among different precipitation regimes for several soil bacteriall populations. These bacteriall genera were defined as the inactive population, thereby indicating a diverse adaptation strategy of different bacterial groups [21].

\section{Conclusion}

This detailed description of bacterial communities in 5 soil water cotent experiment of the West foot of Daxing'an Mountains soil indicates that community structures vary according to the drought stress. Actinobacteria could play an important role in arid soil. In the absence of crops, soil water content has a direct impact on bacterial communities. Next, we will further explore the function of actinomycetes in black soil and find out the beneficial bacteria in drought tolerant bacteria.

\section{Acknowledgment}

This work was supported by the National Key Research and Development Program of China (2019YFC0507600, 2019YFC0507605), the Post doctoral foundation of Inner Mongolia University (5175505), the National Natural Science Foundation of China (31860356), The Major Project of Science and Technology in Inner Mongolia of China(2019ZD009, zdzx2018017) and the Leading Talent Project of "Grassland Talents" in Inner Mongolia of China.

\section{References}

1. Nijssen, B, O'donnell, G. M, Hamlet, A. F., and Lettenmaier, D. P. Hydrologic sensitivity of global rivers to climate change. Clim. Change 50,143-175 (2001).

2. Su, B, Xiao, B., Zhu, D, and Jiang, T. Trends in frequency of precipitation extremes in the $\mathrm{Y}$ angtze River basin, China: 1960-2003. Hydrol. Sci. J. 50, 479-492 (2005).

3. Li, Y, Adams, J, Shi, Y, Wang, H, He, J-S, and Chu, H. Distinct Soil Microbial Communities in habitats of differing soil water balance on the Tibetan Plateau. Sci. Rep. 7:46407 (2017).

4. Dao Zhang, Baorui Chen, Xiaoping Xin. Variation characteristics of temperature and precipitation patterns in Hulunbeier grassland from 1960 to 2015 $[\mathrm{J}]$. Agricultural resources and Regionalization in China, 39 (12):126-133 (2018).

5. Yinyin Liu, Feng Li, QinyeSun. esearch progress of soil microorganism in wetland ecosystem[J] . Journal of applied and Environmental Biology, 19(3): 5 47-552 (2013).

6. Lupatini, M, Suleiman, A.K.A, Jacques, R.J.S., Lemos, L.N, Pylro, V.S., Van Veen, J.A., Kuramae, E.E, and Roesch, L.F.W. Moisture is more important than temperature for assembly of both potentially active and whole prokaryotic communities in subtropical grassland. Microb. Ecol. 77, 460-470 (2019).

7. Meisner, A, Jacquiod, S, Snoek, B. L, Ten Hooven, F. C, and V an Der Putten,W. H. Drought legacy effects on the composition of soil fungal and prokaryote communities. Front. Microbiol. 9:294. (2018).

8. Stres, B, Danevcic, T., Pal, L, Fuka, M.M., Resman, L, Leskovec, S, Hacin, J, Stopar, D., Mahne, I, and Mandic-Mulec, I. Influence of temperature and soil water content on bacteriall, archaeal and denitrifying microbial communities in drained fen grassland soil microcosms. FEMS Microbiol. Ecol. 66, 110122(2008).

9. Muhammad Umair, Ningxiao SunHongmei Du, Nan Hui, Muhammad Altaf, Baoming Du Shan Yinand Chunjiang Liu, Bacteriall Communities Are More Sensitive to Water Addition Than Fungal Communities Due to Higher Soil $\mathrm{K}$ and $\mathrm{Na}$ in a Degraded Karst Ecosystem of Southwestern China. Front. Microbiol. 11:562546 (2020).

10. Supramaniam, Y, Chong, C.W, Silvaraj, S, and Tan, I.K.P. Effect of short term variation in temperature and water content on the bacteriall community in a tropical soil. Appl. Soil Ecol. 107, 279-289 (2016).

11. Lupatini, M, Suleiman, A.K.A., Jacques, R.J.S, Lemos, L.N, Pylro, V.S., Van Veen, J.A, Kuramae, E.E, and Roesch, L.F.W. Moisture is more important than temperature for assembly of both potentially active and whole prokaryotic 
communities in subtropical grassland. Microb. Ecol. 77, 460-470 (2019).

12. Hermans S M, Buckley H L, Case B S, et al. Using soil bacteriall communities to predict physicochemical variables and soil quality $[\mathrm{J}]$. Microbiome, 8(1) (2020).

13. Na X, Yu H, Wang P, et al. Vegetation biomass and soil moisture coregulate bacteriall community succession under altered precipitation regimes in a desert steppe in northwestern China[J]. Soil Biology and Biochemistry, 136:107520 (2019).

14. Zhang $\mathrm{B}$, Wu $\mathrm{X}$, Tai $\mathrm{X}$, et al. Variation in Actinobacteriall Community Composition and Potential Function in Different Soil Ecosystems Belonging to the Arid Heihe River Basin of Northwest China[J]. Frontiers in Microbiology, 10:2209 (2019).

15. Berendsen, E.M, Boekhorst, J, Kuipers, O.P., WellsBennik, M.H, A mobile genetic element profoundly increases heat resistance of bacteriall spores. The ISME Journal 10, 2633-2642(2016).

16. Fierer, N, Lauber, C.L, Ramirez, K.S, Jesse, Z, Mark, A.B, Rob, K, Comparative metagenomic, phylogenetic and physiological analyses of soil microbial communities across nitrogen gradients. The ISME Journal 6, 1007-1017(2012).

17. Bell, C.W, Tissue, D.T, Loik, M.E, Wallenstein, M.D, Acosta-Martinez, V, Erickson, R.A., Zak, J.C, Soil microbial and nutrient responses to seven years of seasonally altered precipitation in a Chihuahuan Desert grassland. Global Change Biology 20, 16571673(2014).

18. Sorensen, P.O, Germino, M.J, Feris, K.P, Microbial community responses to 17 years of altered precipitation are seasonally dependent and coupled to co-varying effects of water content on vegetation and soil C. Soil Biology and Biochemistry 64, 155163(2013).

19. Cregger, M.A, Schadt, C.W, McDowell, N.G, Pockman, W.T, Classen, A.T, Response of the soil microbial community to changes in precipitation in a semiarid ecosystem. Applied and Environmental Microbiology 78, 8587-8594 (2012).

20. Zeng, Q, Brown, P.H, Soil potassium mobility and uptake by corn under differential soil moisture regimes. Plant and Soil 221, 121-134 (2000).

21. Zhang, X, Wei, H. W, Chen, Q. S, and Han, X. G. (2014b). The counteractive effects of nitrogen addition and watering on soil bacterial communities in a steppe ecosystem. Soil Biol. Biochem. 72, 2634 (2014).

22. Francioli D, Schulz E, Buscot F, et al. Dynamics of Soil Bacterial Communities Over a Vegetation Season Relate to Both Soil Nutrient Status and Plant Growth Phenology[J]. Microbial Ecology, (2018).

23. Zhalnina, K, Louie, K. B, Hao, Z, Mansoori, N, Rocha, U. N, Shi, S, et al. Dynamic root exudate chemistry and microbial substrate preferences drive patterns in rhizosphere microbial community assembly. Nat. Microbiol. 3,470-480 (2018).

24. Hayakawa, M. Studies on the isolation and distribution of rare actinomycetes in soil. Actinomycetologica 22, 12-19 (2008).

25. Hill, P, Krištùfek, V, Dijkhuizen, L, Boddy, C, Kroetsch, D, and van Elsas, J. D. Land use intensity controls actinobacterial community structure. Microb. Ecol. 61, 286-302 (2011).

26. Arocha-Garza, H. E, Castillo, R. C, Eguiarte, L. E, Souza, V, and Torre-Zavala, S. D. High diversity and suggested endemicity of culturable Actinobacteria in an extremely oligotrophic desert oasis. PeerJ 5:e3247 (2017).

27. Eilers, K. G, Lauber, C. L, Knight, R., and Fierer, N. Shifts in bacterial community structure associated with inputs of low molecular weight carbon compounds to soil. Soil Biol. Biochem. 42, 896-903 (2010). 